\title{
Investigación del Trastorno Formal del Pensamiento en la esquizofrenia: una mirada crítica
}

RESUMEN: Se examina parte de la investigación más reciente realizada en torno al Trastorno Formal del Pensamiento en esquizofrenia para a demostrar que un obstáculo significativo ha sido la manera en que estos "síntomas mentales" han sido concebidos, en particular su status ontológico y epistemológico.

PALABRAS CLAVE: Trastorno Formal del Pensamiento, Esquizofrenia, Análisis crítico.
ABSTRACT: Recent research upon the group of "mental symptoms" called Formal Thought Disorder in schizophrenia is reviewed. Assumptions concerning their ontological and epistemological status are criticized as the key factors hindering progress for understanding the phenomena.

KEY WORDS: formal thought disorder, schizophrenia, conceptual analysis.

\section{Introducción}

Pese a la gran cantidad de investigación $(1,2,3,4,5)$ llevada a cabo por autores como E. Bleuler (6), Jung (7), Vygotsky (8), Schilder (9), Piro (10), o Goldstein (11), la comprensión de los síntomas denominados 'Trastorno Formal del Pensamiento' (TFP) ha avanzado escasamente $(12,13)$. Aquí se propondrá que esto es consecuencia, en grado importante, de problemas conceptuales y se examinará parte de la investigación más reciente a objeto de ilustrar dicha proposición.

El TFP, al que Bleuler confirió centralidad en su concepto de esquizofrenia (6), es un síntoma frecuente en dicha condición. En sus grados leves, su frecuencia varía entre el $65 \%$ y el $90 \%(14,15)$, y en grados severos llega a afectar al $45 \%$ los de pacientes (16). Además, se asocia con recaídas (17) y con peor adaptación laboral (18), punto rebatido por Huber et al (19) y Parnas (20).

Pese a que se ha avanzado significativamente en el nivel de confiabilidad entre evaluadores para diagnosticar el TFP, aún no entendemos bien qué es, y porqué y cómo se diagnostica. En esto, el TFP es similar a otros procesos cognitivos aparentemente simples pero difíciles de estudiar, como el reconocimiento de patrones visuales (21).

Con el paso del tiempo, se han acumulado numerosas metáforas inspiradas por sucesivas teorías psicológicas y, de no mediar un exámen conceptual crítico, esta tendencia continuará. Específicamente, las definiciones del TFP como 'un tipo de habla dificil de entender', ya sea porque es 'confusa', 'desorganizada', 'idiosincrática', o 
'bizarra', resultan inadecuadas para la investigación neurobiológica y neurocognitiva, pues dichas definiciones aluden a una 'dificultad de entender' que tiene lugar en el fuero interno de quién lo diagnostica, y no en la mente/cerebro del paciente. Aunque retornaremos a esto más abajo, adelantaremos aquí el punto que si se define el TFP como 'habla dificil de entender', entonces la investigación con neuroimágenes, psicofisiológica, o neuropsicológica está correlacionando rasgos de un fenómeno mental del interlocutor (por ejemplo, un puntaje de desorganización conceptual en el BPRS) con datos que reflejan lo que ocurre en el cerebro/mente del paciente (por ejemplo, una potencial evocado).

La necesidad de una revisión crítica en esta área tambien deriva del hecho que la literatura que aborda la rehabilitación de los pacientes con TFP es escaza $(22,23$, $24,25,26)$ y se necesita un modelo del lenguaje en la esquizofrenia que considere factores neurobiológicos, psicológicos, linguísticos, interaccionales y culturales. La construcción de dicho modelo requiere de un concepto de TFP apropiado para dicha integración.

\section{El objeto de estudio}

En este artículo abordaremos el "habla desorganizada" (27) que se observa en los sujetos con el diagnóstico de esquizofrenia. Por ende, no se abordarán anomalías observadas en trastornos afectivos, ni con delirios, obsesiones, o cuadros disfásicos. Restringimos nuestro análisis, a diferencia de otros $(28,29,30)$, a una sola entidad nosológica, pues no es posible descartar a priori que la enfermedad en que ocurre el TFP tenga efectos patogénicos además de patoplásticos sobre este grupo de síntomas.

\section{TFP: la visión recibida (VR)}

El modelo tradicional del TFP puede ser resumido en los siguientes enunciados: 1. El TFP es un fenómeno en el pensamiento/lenguaje del paciente; 2 . Es fundamental y específico de la esquizofrenia; 3. Es un fenómeno homogéneo; 4. Deriva de un único mecanismo causal. 5. Es un fenómeno estable.

En efecto, para Bleuler (6) el TFP era la alteración fundamental desde la cual todos los sintomas de la esquizofrenia derivaban. Este concepto ha persistido durante todo el siglo veinte permeando aún las visiones más heterodoxas (31). Actualmente, los textos describen al TFP de un modo somera: 'aquello de lo que un sujeto habla es el contenido y el modo en que lo habla es la forma' $(32,33)$. Los delirios ejemplificarian la patología del 'contenido' mientras que el TFP reflejaría patología de la 'forma'. En su versión actual, la VR asume a) que el delirio deriva de una disfunción mental 'central' mientras que el TFP refleja una disfunción linguística o 'local' y b) que 'forma' y 'contenido' del pensamiento y del lenguaje son dimensiones independientes. Aunque no abordaremos esto en más detalle, baste decir que ambos implícitos son debatibles en psicopatología así como en la filosofía de la mente y del lenguaje $(34,35,36,37,38,39)$. A continuación se intentará mostrar que la VR aún es muy influyente. 


\section{Investigación empirica}

Los investigadores se han abocado a la búsqueda de la "causa" del TFP pues han asumido que la descripción de las anormalidades comunicativas observables en la esquizofrenia es una tarea ya concluida. Sin embargo, no existe una descripción psicopatológica que pueda reclamar ser la 'final', aún cuando se la consagre como oficial en los manuales diagnósticos. Hay una rica diversidad de descripciones en diferentes tradiciones psicopatológicas cuya análisis comparativo permanece inexplorado (por ejemplo, 40,41, 42, 10,43,31). Tampoco se dispone de información descriptiva sobre qué dominios conductuales son afectados por el TFP, qué areas temáticas son comprometidas, o si el TFP adopta ciertas constelaciones sincrónicas y diacrónicas.

La idea de relacionar el TFP con deficits cognitivos ya había estado presente en el pionero trabajo "neuropsicológico" de Masselon en 1902 (47). Más tarde se consideró al TFP ya sea como resultante de una ineficiente "administración" de recursos cognitivos o bien de una anormalidad de las "representaciones mentales". Ejemplos de la primera perspectiva, "disejecutiva", fueron Goldstein (11) y Cameron (48). Ejemplos de la segunda perspectiva, "disemántica", fueron los trabajos de Piro (10) sobre el aumento y distorsión del halo semántico de las palabras y de Bannister (49) sobre la estabilidad y coherencia de constructos.

A continuación se examinarán tres líneas actuales de investigación: En primer lugar, los trabajos que relacionan el TFP con anormalidades en la representación neural del significado, ya sean modelos basados en los conceptos de las 'redes semánticas' (44) como aquellos basados en la neuropsicología clásica. En segundo lugar, revisaremos el trabajo que vincula al TFP con las funciones ejecutivas (45). En tercer lugar, se examinará la investigación que concibe el TFP como una falla en la producción de un discurso cohesionado (46).

\section{TFP como excesiva activación semántica}

Las ideas subyacentes a esta aproximación se pueden resumir del modo siguiente: a) El conocimiento que los sujetos tienen del mundo se almacena en la forma de redes formadas por "nodos" interconectados a través de "vínculos" de intensidad variable, b) El 'curso del pensamiento' depende del automático flujo asociativo en dichas redes, c) El TFP resultaría de una excesiva difusión de la activavidad neural. Es destacable que la evidencia indique que dicha anormalidad semántica ocurriría en el hemisferio cerebral izquierdo, dominante para el lenguaje (50). Este modelo que parece en una primera instancia la implementación biológica de la VR bleuleriana, sin embargo amerita varios comentarios.

En primer lugar, varios estudios indican que, o bien el TFP resulta de un priming semántico reducido, o bien no se asocia con anormalidad alguna del 'priming' semántico $(51,52,53,54,55,56,57,50)$. Datos tan contrapuestos podrían resultar de problemas 
metodológicos como el efecto de la medicación, la duración de la enfermedad, respuestas lentas de los pacientes $(58,59)$, y el hecho que mínimas modificaciones experimentales pueden gatillar la participación de procesos cognitivos 'estratégicos' $(60,52,55)$. En segundo lugar, la descripción asociacionista del pensamiento y el lenguage no está exenta de críticos $(61,62,36,5)$, en el sentido que quizás no captura el carácter connotativo y la pragmática del lenguaje humano (63), aspectos importantes del TFP en la esquizofrenia (64). En tercer lugar, si bien el priming semántico excesivo podría explicar la 'laxitud asociativa', no es fácil entender cómo los otros síntomas del TFP se derivarían a partir del mismo defecto. Por último, la noción de que la excesiva activación semántica explicaría el que los pacientes con esquizofrenia en general y con TFP en particular exhiban reducida "sensibilidad al contexto linguístico" (65), ha sido rebatida y se ha propuesto que otros factores estarían en juego (62).

\section{El TFP como déficit semántico}

McKenna y colaboradores en Cambridge han investigado el estado del sistema semántico de sujetos con esquizofrenia utilizando pruebas semánticas no-automáticas y han encontrado que el TFP 'positivo', que abarca algunos pero no todos los síntomas clásicos de TFP, se ha asociado con déficits en el 'sentido común', en la capacidad para definir palabras, y con errores semánticos en el discurso $(66,67,68,69)$. El TFP tambien estaría asociado con déficits en pruebas de fluidez verbal semántica y de asociación entre palabras y figuras (70). El hecho que el rendimiento en pruebas de denominación de figuras esté relativamente preservado se ha interpretado como indicativo de que el déficit semántico en el TFP ocurriría a nivel "central" $(70,71)$.

Un problema con este enfoque es que el discurso de los sujetos con demencia semántica o de pacientes con enfermedad de Alzheimer y déficit semántico no corresponde al cuadro de TFP observado en la esquizofrenia. Es decir, un déficit semántico parece ser insuficiente por sí solo para generar TFP.

\section{TFP como disfunción ejecutiva del lenguaje}

Se ha denominado "funciones ejecutivas" a rutinas cognitivas propias de la conducta orientada a metas, tales como la formación de intenciones, la planificación e iniciación de conductas, la corrección de errores, la manipulación de información abstracta, etc $(72,73,74,75,76)$. Los sujétos que exhiben déficits en algunas de estas rutinas son referidos como afectados de un 'sindrome disejecutivo' (77); . usaremos el término sin adentrarnos en el debate respecto de su status y estructura interna $(78,79,80)$.

McGrath ha propuesto que el TFP resulta de un 'sindrome disejecutivo' que afecta la producción de lenguaje $(81,45,29)$. Este autor encontró que TFP 'positivo' se asociaba con el fenómeno de 'pérdida del set cognitivo en curso' (Stroop [interferencia]); 
el síntoma 'pobreza del habla' se asociaba con la inhabilidad para 'establecer un set cognitivo' (Wisconsin [categorías]); y el síntoma 'perseveración' se asociaba con la inhabilidad para 'alternar entre sets cognitivos' (Trail Making Test [AB]). Otros autores también han sugerido que el TFP resulta de un trastorno de las funciones ejecutivas $(30,82,12,83,13,84,85,86)$.

Existen aquí varios problemas metodológicos. En primer lugar, se ha cuantificado el TFP utilisando diferentes instrumentos psicopatológicos, de los cuales se asume, sin una base empírica, que capturan las mismas conductas. Aún en los casos en que se usa el mismo instrumento psicométrico, diferentes trabajos obtienen los puntajes de TFP sumando puntajes de distintos síntomas. Del mismo modo, las escalas psicopatológicas son aplicadas de manera diversa, en entrevistas que varían en duración y contenido. Esto último es importante porque la detección de síntomas infrecuentes depende de la extensión de la muestra conductual obtenida y porque es posible que ciertos temas generen más y distintos tipos de TFP que otros temas. En segundo lugar, las variables neuropsicológicas han sido quantificadas de manera disímil, e incluso cuando los tests usados han sido los mismos, el procedimiento de administración ha cambiado. Finalmente, algunos de estos trabajos han utilizado muestras clínicamente heterogéneas $(100,117)$, lo que cuestiona su relevancia para la esquizofrenia.

Por último y no menos importante, si bien los pacientes neurológicos con síndrome disejecutivo pueden exhibir anormalidades no afásicas del lenguaje, estos pacientes no exhiben el TFP de tipo "esquizofrénico" $(87,88,89)$, lo que sugiere que el síndrome disejecutivo no es suficiente, por sí solo, como factor etiológico del TFP.

\section{TFP como falla de la cohesión en el discurso}

Este enfoque dará pábulo para ilustrar cómo la naturaleza del fenómeno psicopatológico cambia de acuerdo a la teoría con que se le aborda.

Rochester \& Martin (4), autores 'fundacionales' de esta aproximación, señalaron la necesidad de que el lenguage de los pacientes fuese analizado de una manera más 'natural'. Se habia hecho evidente que visiones conductistas del lenguaje o cuantificaciones como el análisis de la complejidad sintáctica, la razón 'type-token', o el método de Cloze, ignoraban la esencia del discurso, es decir, su significado (89).

En esta aproximación el TFP ha sido redefinido como un déficit del hablante para generar cohesión en el discurso $(90,91,13)$. El procedimiento consiste en la grabación y transcripción del habla del paciente, y la cuantificación del tipo de vínculos cohesivos utilisados en el texto. Dicho análisis es llevado a cabo por sujetos que deberían carecer de información sobre los interlocutores y su contexto. Subsecuentemente, los resultados son correlacionados con medidas de atención, capacidad de abstracción, etc. 
El enfoque "discohesivo" ignora tanto el contexto en que las verbalizaciones fueron emitidas como la necesidad de "expandir el texto" cuando se analizan segmentos de discurso (92). Esto es importante pues el contexto es esencial para determinar la incoherencia de las verbalizaciones de un sujeto. Así mismo, centrarse meramente en la cohesión textual es estrecho pues ella no garantiza, por sí sola, la coherencia del discurso. Esta última tiene lugar a un nivel más conceptual y es parcialmente construida por el interlocutor $(93,94)$. En otras palabras, la coherencia de una conversación no depende de la relación entre las verbalizaciones emitidas, sino que de la relación entre los 'actos de habla' realizados con dichas verbalizaciones (92).

Además que las fallas de cohesión en el discurso no permiten diferenciar el habla de sujetos con esquizofrenia de la de sujetos normales (95), la validez de este enfoque es también cuestionada por el hecho de que los pacientes que exhiben fallas de cohesión en el discurso tras haber sufrido daño cerebral no exhiben TFP de tipo "esquizofrénico" (96). En la misma línea, los pacientes afásicos con dafio al hemisferio derecho o con demencia (97) presentan déficits macro-estructurales en su discurso pero no exhiben TFP de tipo "esquizofrénico".

\section{Revisiones efectuadas a la Visión Recibida (VR)}

En la medida que el lenguaje de los pacientes ha sido estudiado en detalle, se han cuestionado la especifidad y estabilidad del TFP así como su centralidad para la esquizofrenia.

\section{El TFP es inespecifico}

La opinión de Kurt Schneider (98) de que el TFP carecía de toda especificidad ha sido refrendada por autores más recientes quienes han encontrado que el TFP también ocurre en una minoría significativa de sujetos sin patología psiquiátrica, en individuos con trastornos de la personalidad, trastornos afectivos psicóticos o no psicóticos, y en el tratomo esquizo-afectivo $(14,88,15,99,100,101,102,16,1,103,104,105,106,107$, $108,109)$. Aunque estos hallasgos dependen de la validez y fiabilidad de los instrumentos con que se obtuvieron (110), la evidencia es significativa. Pese a ello, autores como Holzman (111) hablan de "la falla fundamental única" en la esquizofrenia, mientras que otros aún consideran al TFP como patognomónico (el síndrome esquizofrénico axial) (112).

\section{La heterogeneidad del TFP}

No todos los sujetos diagnosticados con TFP exhiben el mismo tipo de conductas linguísticas $(113,114)$ y, más aún, no es infrecuente que dos pacientes con TFP presenten anormalidades linguísticas y comunicativas muy distintas. En un intento por dar cuenta de dicha heterogeneidad, Andreasen $(14,88)$ redefinió el TFP como trastornos del pensamiento, lenguage y de la comunicación y construyó la escala del mismo nombre 
(TLC) que contiene 18 síntomas (115). Testimonian también la heterogeneidad del TFP el Indice de Desorden del Pensamiento (1), de 36 categorías, el Indice de Pensamiento Bizarro e Idiosincrático (116), que contiene 10 categorías, y la escala CLANG (94) que consta de 17 síntomas. Además, el análisis de algunos de estos instrumentos ha revelado una estructura factorial heterogenea $(117,118,24,119)$.

La variedad de descripciónes clásicas de síntomas linguísticos, cuya correspondencia permanece indeterminada, indican también que el TFP es heterogéneo. Nos referimos aquí, por ejemplo, a las descripciones de Kussmaul (120), Séglas (43), Kraepelin (121, 122), Carl Schneider (citado en 3, 123, 124), Lecours (42), o Kleist $(125,126)$.

\section{TFP: un fenómeno inestable}

Es posible observar que algunos pacientes exhiben fluctuaciones significativas en el grado de incoherencia en su lenguaje, aún en el corto plazo. El lenguaje de otros pacientes es más bien establemente incomprensible. En otros, la intensidad del TFP puede variar de acuerdo a la situación social en que la persona está participando. El TFP es también impredecible, es decir, no se asocia sistemáticamente con rasgos específicos del discurso, o con ciertos temas o situaciones $(1,127)$. En particular, se requiere más observación respecto de la relación entre TFP y temas afectivamente significativos y/o delirantes. En el largo plazo, sabemos que el TFP 'positivo' tiende a ser menos frecuente en pacientes de mayor edad, pero dicha tendencia presenta notables excepciones observables en la práctica clínica. Además, las categorias descriptivas, el TFP como habla fluente y difícil de entender, son demasiado globales $(128,129,130)$.

\section{¿Qué tipo de entidad es el TFP?}

Entonces, el TFP parece ser heterogéneo, inespecífico, e inestable, y el DSM-IV ha modificado la VR para incorporar estas carecterísticas. Sin embargo, los dos supuestos básicos de la VR, que denominaremos 'ontológico' y 'epistemológico', permanen sin ser cuestionados, pese a que han impedido que el uso de metodologias 'duras' (por ejemplo, potenciales relacionados a eventos o neuroimágenes) produzcan avances sustanciales en la comprensión del TFP.

\section{Una nota histórica}

Hacia fines del siglo XIX un cúmulo de síntomas del habla, la escritura y de la expresión artística habían sido descritos por Falret, Séglas, Morselli o Kussmaul. Entonces, tuvo lugar una selección de algunos de los síntomas que formaban parte del "lenguaje de los insanos", y ciertos síntomas fueron incluídos y otros excluídos (por ejemplo, las alteraciones sintácticas). Dicha selección (131) fue determinada más por el marco conceptual asociacionista y de la 'etología del pensamiento' $(132,133,134)$ y menos por datos empíricos, los cuales tendían a contradecir a la emergente VR 
$(135,136)$. Los elementos que fueron seleccionados pasaron a constituir dicha visión recibida, la que gradualmente fué adquiriendo una suerte de 'autonomía', como se observa en el simposio sobre lenguaje y pensamiento en la esquizofrenia organizado por Kasanin (137).

\section{Ontologia del TFP}

El TFP no existe en forma independiente en la naturaleza y fuera del ámbito del lenguaje de los interlocutores. Su status de existencia es diferente al de una piedra o un planeta, entidades cuyo "descubrimiento" no agrega nada a su existencia. Por el contrario, el TFP es un constructo que opera como una 'vía final común' en virtud de la cual diferentes tipos de conductas lingúísticas son evaluadas por un interlocutor socialmente habilitado. La cualidad de ser "formalmente desordenadas" no es una propiedad inherente e invariante de las palabras utilisadas por el paciente si no que es una construcción basada en el contexto linguístico y socio-cultural, en las características del interlocutor, y en claves derivadas de lo verbalizado por el sujeto, que permiten o impiden atribuirles coherencia a los actos de habla realizados por el hablante por medio de sus palabras, gestos, $\mathrm{y}$ afectos movilizados.

Ignorar lo recién expuesto ha llevado ha creer que el grupo de conductas a que se refiere el TFP es estable e independiente de la teoría utilizada para describirlo, como se las diferentes teorías se refieren al mismo fenómeno. Sin embargo, las sucesivas redefiniciones propuestas (por ejemplo, "pensamiento concreto", "sobre-inclusivo", "disemántico", etc) no son equivalentes. Cada aproximación teórica selecciona cuales conductas han de ser consideradas en la construcción del fenómeno y cuales no deben ser tomadas en cuenta $(138,139)$. Por supuesto, estas diferencias en la construcción del síntoma repercuten directamente en los resultados de la investigación. Ilustrativo aquí resulta el debate sobre la heredabilidad del TFP $(140,141,142,143)$, donde se ha discutido "qué es" el TFP sin que se llege a acuerdo. Lo que ocurre es que cada teoría determina la inclusión y descripción de ciertos ítems y esto, a su vez, determina las fronteras del fenómeno. En suma, el TFP no puede ser "cuantificado" de un modo teóricamente neutral (144).

\section{Epistemología del TFP}

El TFP no contiene toda la información necesaria para ser identificado como tal en cualquier circunstancia (145). Por el contrario, cuando el clínico diagnostica el habla de un sujeto como 'desorganizada', lo hace influido por variados factores tales como el hecho que la persona en cuestión presenta psicopatología adicional, que las verbalizaciones fueron emitidas en una entrevista que ha tenido lugar, por ejemplo, en un hospital y no durante un recital de poesía o un servicio religioso. Además, el juicio de que el discurso de un individuo es incomprehensible depende fuertemente de rasgos estables del clínico, como su formación general, tolerancia a significados ambiguos, familiaridad con el paciente, entrenamiento psicoterapéutico (146), así como de características transitorias, como su nivel de alerta, humor, o tiempo disponible para la entrevista. 


\section{Qué concepto utilizar?}

Los conceptos en psicopatología tienen necesariamente un importe teórico. Por ejemplo, el concepto de 'habla desorganizada' del DSM-IV, sugiere que el problema que se observa en estos pacientes afecta predominantemente la 'facultad' o 'módulo' del lenguaje. Por otro lado, el concepto de TFP hace referencia a un trastorno cognitivo más amplio no limitado a la 'facultad' del lenguage, lo cual es útil pues permite que el tema de si el trastorno es del lenguaje y/o del pensamiento y/o de toda la conducta sea investigado empíricamente. El concepto de TFP también enfatiza los aspectos "formales", otro término opaco, de los actos de habla (147), en oposición a su contenido proposicional. Esto sugiere la necesidad de estudiar anormalidades de los procesos procedurales del pensamiento y del habla descritos por Levelt $(36,148)$.

En definitiva, el dilema no es elegir entre conceptos depuradamente descriptivos y ateóricos y otros conceptos teóricos inevitablemente sesgados. Más bien la tarea es utilizar los conceptos mas útiles para la tarea a realizar, fundamentando en forma explícita la eleción de uno u otro concepto. En este sentido, la noción de TFP continúa siendo útil en la medida que se hagan explícitos los supuestos que le son propios.

\section{Conclusiones}

1.- Se ha criticado de la psicopatología no discutir abiertamente los supuestos conceptuales en que basa su práctica (149). Aquí se ha intentado exponer algunos de los conceptos que subyacen a la investigacion del TFP en esquizofrenia.

2.- La validez de la noción de TFP es cuestionable pese a que en su diagnóstico se haya logrado una alta confiabilidad entre evaluadores. Como se sabe, la fiabilidad es necesaria pero no suficiente para que un constructo sea válido $(150,151)$.

3.- La evaluación del discurso de otro individuo ocurre en la mente de quién escucha dicho discurso y es lógicamente independiente de lo que ocurre en la mente de quién lo emite. Por tanto, cuando se correlaciona un "puntaje" de TFP con una variable 'dura' como una activación cerebral medida por PET, se está observando la asociación entre una magnitud que depende de eventos mentales del interlocutor con otra magnitud que refleja la activación que tiene lugar en el cerebro del hablante (152).

4.- El DSM-IV refuerza el implícito de que el TFP es un fenómeno autónomo precisamente porque se declara 'ateórico' e incorpora una revisión parcial de la VR. Así genera la impresión de que es una constatación transparente de las cosas 'tal como son' y, en consecuencia, sus supuestos teóricos permanecen ocultos. Sin embargo, la realidad de la comunicación y del lenguaje anormal es bastante más compleja. 
ORIGINALES Y REVISIONES

\section{BIBLIOGRAFIA}

1. Johnston M H, Holzman P S. (1979) Assessing schizophrenic thinking. A clinical and research instrument for measuring thought disorder. San Francisco, Jossey-Bass.

2. Maher B. (1972) "The Language of Schizophrenia: A Review and Interpretation", British Journal of Psychiatry, 120, 3-17.

3. Reed J L. (1970) "Schizophrenic Thought Disorder: a Review and Hypothesis". Comprehensive Psychiatry, Vol. 11, No. 5, 403-432.

4. Rochester, S.R., Martin, J.R. (1979): Crazy talk: a study of the discourse of schizophrenic speakers, Plenum Press, New York.

5. Schwartz, S. (1978): "Do schizophrenics give rare word association?" Schizophrenia Bulletin, 4, 2: 248-271.

6. Bleuler, E. (1911): Demencia precoz. El grupo de las esquizofrenias, Hormé, Buenos Aires ( $1^{\mathrm{a}}$ edición alemana de 1911 , la traducción española procede de la edición en lengua inglesa de 1950).

7. Jung C G. (1907) "The Psychology of Dementia Praecox", en The Collected Papers of C. G. Jung, Vol. 3, Bollinger Series XX, New Jersey, Princeton University Press.

8. Vygotsky L. (1934) "Thought in schizophrenia". Archives of Neurology and Psychiatry, 31: 1063-1077.

9. Schilder, P. (1920): “On the development of thoughts", Z. Neurol. Psychiat, 59: 250-263, en D. Rapaport, The Organization and Pathology of Though, Columbia University Press, New York [1951].

10. Piro, S. (1987): El lenguaje esquizofrénico, Fondo de Cultura Económica, México.

11. Goldstein K. (1944) "Methodological Approach to the study of Schizophrenic Thought Disorder", in Kasanin J S (ed.) Language and Thought in Schizophrenia. New York, The Norton Library.

12. Barch, D. M., Berenbaum, H. (1996): "Language production and thought disorder in schizophrenia", Journal of Abnormal Psychology, 105, 1: 81-88.

13. Docherty N M, Hawkins K A, Hoffman R E, Quinlan D M, Rakfeldt J, Sledge W H. (1996b) "Working Memory, Attention, and Communication Disturbances in Schizophrenia". Journal of Abnormal Psychology, Vol. 105, No. 2, 212-219.

14. Andreasen, N.C. (1979a): "Thought, Language and Communication Disorders. I. Clinical assessment, definition of terms, and evaluation of their reliability". Archives of General Psychiatry, 36: 1315-1321.

15. Andreasen N C and Grove W M. (1986) "Thought, Language, and Communication in Schizophrenia: Diagnosis and Prognosis", Sçhizophrenia Bulletin, Vol. 12, No. 3: 348-359.

16. Marengo J T \& Harrow M. (1997) "Longitudinal Courses of Thought Disorder in Schizophrenia and Schizoaffective Disorder", Schizophrenia Bulletin, Vol. 23, No.2, 273-285.

17. Wilcox, J. A. (1990): "Thought disorder and relapse in schizophrenia", Psychopathology, 23: 153-156.

18. Rancenstein J M, Penn D, Harrow M \& Schleser R. (1999) "Thought Disorder and Psychosocial Functioning in Schizophrenia", The Journal of Nervous and Mental Disease, Vol. 187, No. 5, 281-289.

19. Huber, G., Gross, G., Schuttler, R., Linz, M. (1980): “Longitudinal studies of schizophrenic patients", Schizophrenia Bulletin, 6, 4: 592-605. 
20. Parnas, J., Jorgensen, A., Teasdale, T. W., Schulsinger, F., Mednick, S. A. (1988): "Temporal course of symptoms and social functioning in relapsing schizophrenics: a 6-year follow-up", Comprehensive Psychiatry, 29, 4: 361-371.

21. Bechtel W. (1994) "Connectionism", in Guttenplan S (Ed.), 'A Companion to the Philosophy of Mind', Oxford, Blackwell.

22. Benedetti G and Furlan P M. (1993) The Psychotherapy of Schizophrenia. Effective Clinical Approaches-Controversies, Critiques and Recommendations, Toronto, Hogrefe \& Huber Publishers.

23. Birchwood M \& Tarrier N. (1994) Psychological Management of Schizophrenia. Chichester, Wiley.

24. Chen E Y H, Lam L C W, Kan C S, Chan C K Y, Kwok C L, Nguyen D G H \& Chen R Y L. (1999) "Language Disorganisation in Schizophrenia: Validation and Assessment With a New Clinical Rating Instrument", Hong Kong Journal of Psychiatry, 6 (1), 4-13.

25. Rosenbaum, B., Sonne, H. (1986): The Language of Psychosis. New York University Press, New York.

26. Walsh I. (1997) "Conversational Skills and Schizophrenia". An exploration, in France J and Muir N (Eds.), Communication and the Mentally Ill Patient. Developmental and Linguistic Approaches to Schizophrenia. London, Kingsley Publishers.

27. American Psychiatric Association. (1994) DSM-IV: Diagnostic and Statistical Manual of Mental Disorders. 4th ed. Washington, DC: APA Press.

28. Berrios, G.E. (1996):The history of mental symptoms, Cambridge University Press, Cambridge, pp. 71-84.

29. McGrath J, Hengstberger P, Scheldt S, and Dark F. (1997) "Thought Disorder and Executive Ability". Cognitive Neuropsychiatry, 2 (4), 303-314.

30. Persons J B, Baron J. (1985) "Processes Underlying Formal Thought Disorder in Psychiatric Inpatients". The Journal of Nervous and Mental Disease, Vol. 173,No. 11: 667-676.

31. Wróbel J. (1990) Language and Schizophrenia, Amsterdam, Philadelphia, John Benjamin Publishing Company.

32. Gelder, M., Gath, D. y Mayou, R. (1997): Oxford Textbook of Psychiatry. 2nd ed., Oxford University Press, Oxford.

33. Sadock B J. (2000) "Signs and Symptoms in Psychiatry", in Sadock B J \& Sadock V A (Eds.), Kaplan and Sadock's Comprehensive Textbook of Psychiatry, Philadelphia, Lippincott Williams \& Wilkins.

34. Guttenplan, S. (1994): "Syntax/Semantics", en S. Guttenplan (ed.), A Companion to the Philosophy of Mind, Blackwell, Oxford.

35. Johnson-Laird, P. N. (1988): “A taxonomy of thinking”, en R.J. Sternberg y E.E. Smith (eds.), The Psychology of the Human Thought, Cambridge University Press, Cambridge.

36. Levelt W J M. (1989) Speaking. From Intention to Articulation, Cambridge MA, London, The MIT Press.

37. Marslen-Wilson W \& Tyler L K. (1998) "Rules, representations, and the English past tense", Trends in Cognitive Sciences, Vol. 2, No. 11, 428-435.

38. Sperber D \& Wilson D. (1986) Relevance, Communication and Cognition, Oxford, Basil Blackwell.

39. Pinker S. (1994) The Language Instinct: the new science of language and mind, London, Allen Lane, The Penguin Press.

40. Castilla del Pino, C. (1988): "Tipología de los discursos y su utilización en psicopatología", Revista de la Asociación Española de Neuropsiquiatria, 8: 25: 181-194. 
41. Díez, A, Villagrán, J.M., Sánchez, V., Luque, R. (1992): "El discurso psicótico", Psiquiatria Pública, 4, 4: 192-202.

42. Lecours A R. (1995) "Schizophasia: the glossomanic and the glossolalic subtypes", in Sims A (ed.) 'Speech and Language Disorders in Psychiatry'. Gaskell.

43. Séglas J. (1892) Les Troubles du Langage chez les Aliénés, Paris.

44. Spitzer, M. (1997): “A cognitive neuroscience view of schizophrenic thought disorder”, Schizophrenia Bulletin, 23, 1: 29-50.

45. McGrath J. (1996) "The Pathogenesis of Thought Disorder", en Pantelis C, Nelson H E, Barnes T R E (ed.) Schizophrenia. A Neuropsychological Perspective, John Wiley \& Sons.

46. Docherty, N.M., DeRosa, M., Andreasen, N.C. (1996a): "Communication disturbances in schizophrenia and mania", Archives of General Psychiatry, 53: 358-364.

47. Masselon R. (1902) Psychologie des Déments Précoces, Thèse Pour Le Doctorate en Médicine, Faculté de Medicine de Paris.

48. Cameron N. (1944) "Experimental Analysis of Schizophrenic Thinking", in Kasanin J S (ed.). Language and Thought in Schizophrenia. New York, The Norton Library.

49. Bannister D. (1960) "Conceptual Structure in Thought Disordered Schizophrenics". The Journal of Mental Science, Vol. 106, No. 445, 1230-1249.

50. Weisbrod M, Maier S, Harig S, Himmelsbach U and Spitzer M (1998). "Lateralised semantic and indirect semantic priming effects in people with schizophrenia", British Journal of Psychiatry, 172, 142-147.

51. Aloia M S, Gourovitch M L, Missar D, Pickar D, Weinberger DR, Goldberg T E. (1998) "Cognitive Substrates of Thought Disorder, II: Specifying a Candidate Cognitive Mechanism", American Journal of Psychiatry, 155: 1677-1648.

52. Besche C, Passerieux C, Segui J, Sarfati Y, Laurent JP\& Hardy-Baylé MC. (1997) "Syntactic and Semantic Processing in Schizophrenic Patients Evaluated by Lexical-Decision Tasks", Neuropsychology, Vol. 11, No. 4, 498-505.

53. Blum NA \& Freides D. (1995) "Investigating thought disorder in schizophrenia with lexical decision task", Schizophrenia Research, Vol. 16, 217-224.

54. Henik A, Priel B, Umansky R. (1992) "Attention and automaticity in semantic processing of schizophrenic patients", Neuropsychiatry, Neuropsychology and Behavioural Neurology, Vol. 5, No. 3, 161-169.

55. Ober B A, Vinogradov S \& Shenaut G K. (1997) "Automatic Versus Controlled Semantic Priming in Schizophrenia", Neuropsychology, 11, No.4, 506-513.

56. Spitzer M, Braun U, Hermle L \& Maier S. (1993) “Associative Semantic Network Dysfunction in Thought-Disordered Schizophrenic Patients: Direct Evidence from Indirect Semantic Priming", Biological Psychiatry, 34, 864-877.

57. Surguladze, S.A., David, A.S. (1988): "Cognitive neuropsychiatry and schizophrenia", Current Opinion in Psychiatry, 11: 39-44.

58. Barch, D., Cohen, J., Servan-Schreiber, D., Steingard, S., Steinhauer, S., van Kammen, D. (1996): "Semantic priming in schizophrenia: an examination of spreading activation using word pronunciation and multiple SOAs", Journal of Abnormal Psychology, 105: 592-601.

59. Moritz S, Andresen B, Domin F, Martin T, Probsthein E, Kretschmer G, Krausz M, Naber D, \& Spitzer M. (1999) "Increased automatic spreading activation in healthy subjects with elevated scores in a scale assessing schizophrenic language disturbances", Psychological Medicine, 29, 161-170.

60. Bandhari S \& Curtis D. (1998) "Semantic priming in schizophrenia", British Journal of Psychiatry, Vol. 173, 184. 
61. Johnson-Laird, P. N, Herrmann D J, Chaffin R. (1984) “Only Connections: A Critique of Semantic Networks", Psychological Bulletin, 96, No. 2, 292-315.

62. Kuperberg G R, McGuire PK \& David A S. (1998) "Reduced Sensitivity to Linguistic Context in Schizophrenic thought Disorder: Evidence from On-line Monitoring for words in Linguistically Anomalous Sentences", Journal of Abnormal Psychology, Vol. 107, No. 3, 423-434.

63. Forrester M A. (1996) Psychology of Language. A Critical Introduction, London, Sage Publications.

64. Thomas P. (1997) "What Can Linguistic Tell us About Thought Disorder?" In France J and Muir N (Eds.) Communication and the Mentally Ill Patient. Developmental and Linguistic Approaches to Schizophrenia, London, Kingsley Publishers.

65. Spitzer M, Beuckers J, Maier S, Hermle L. (1994) "Contextual insensitivity in schizophrenic patients is due to semantic network pathology: Evidence from pauses in spontaneous speech", Language and Speech, 37, 171-185.

66. Tamlyn D, McKenna J P, Mortimer A M, Lund C E, Hammond S \& Baddeley A D. (1992) "Memory impairment in schizophrenia: its extent, affiliations and neuropsychological character", Psychological Medicine, 22, 101-115.

67. Mortimer A, Corridan B, Rudge S, Kho K, Kelly F, Bristow M \& Hodges J. (1995) "Thought, speech and language disorder and semantic memory in schizophrenia", in Sims A (Ed.) Speech and Language Disorders in Psychiatry, Gaskell.

68. McKenna, P. J. (1994): Schizophrenia and Related Syndromes, Oxford University Press, Oxford.

69. Oh T M, McCarthy R A \& McKenna P J. (1999) "Is There a Language Disorder in Formal Thought Disorder? II. A Study Using the Single Case Approach" (Submitted for Publication).

70. Goldberg T E, Aloia M S, Gourovitch M L, Missar D, Pickar D \& Weinberger D R. (1998) "Cognitive Substrate of Thought Disorder I: The Semantic System", American Journal of Psychiatry, 155: 1671-167.

71. Laws K R, Kondel T K \& McKenna P J. (1999) "A Receptive Language Deficit in Schizophrenic Thought Disorder: Evidence for Impaired Semantic Access and Monitoring", Cognitive Neuropsychiatry, 4 (2), 89-105.

72. Grafman J. (1994) "Alternative frameworks for the conceptualisation of prefrontal lobe functions", in Boller F, Spinnler H \& Hendler J A (Eds.) Handbook of Neuropsychology, Vol. 9, 187-202.

73. Lezak M D. (1995) Neuropsychological Assessment, New York, Oxford University Press.

74. Rabbitt P. (1997) "Introduction: Methodologies and Models in the Study of Executive Function", in Rabbitt P. (ed.) Methodology of Frontal and Executive Function, Hove, UK, Psychology Press.

75. Phillips L H. (1997) Do "Frontal Tests' Measure Executive Function? Issues of Assessment and Evidence from Fluency Tests", in Rabbitt P. (ed.) Methodology of Frontal and Executive Function, Hove, UK, Psychology Press.

76. Robbins T W. (1998) "Dissociating executive functions of the prefrontal cortex", in Roberts A C, Robbins T W, Weiskrantz L. (ed.) The Prefrontal Cortex. Executive and Cognitive Functions, Oxford, Oxford University Press.

77. Wilson B A, Evans J J, Emslie H, Alderman N, Burgess P. (1998) "The development of an Ecologically Valid Test for Assessing Patients with a Dysexecutive Syndrome". Neuropsychological Rehabilitation, 8 (3), 213-228. 
ORIGINALES Y REVISIONES

78. Goldman-Rakic P S. (1998) "The prefrontal Landscape: implications of functional architecture for understanding human mentation and the central executive", in Roberts A C, Robbins T W, Weiskrantz L. (Eds.) The Prefrontal Cortex. Executive and Cognitive Functions, Oxford, Oxford University Press.

79. Parkin A J. (1998) "The central executive does not exist", Journal of the International Neuropsychological Society, 4, 518-522.

80. Baddeley A. (1998) "The central executive: A concept and some misconceptions", Journal of the International Neuropsychological Society, 4, 523-526.

81. McGrath, J. (1991): "Ordering thoughts on thought disorder", British Journal of Psychiatry, 158: 307-316.

82. Harrow M, Lanin-Kettering I, Prosen M, Miller J G. (1983) "Disordered Thinking in Schizophrenia: Intermingling and Loss of Set”. Schizophrenia Bulletin, Vol. 9, 354-367.

83. Barch D M \& Berenbaum H. (1997) "The effect of language production manipulations on negative thought disorder and discourse coherence disturbances in schizophrenia", Psychiatry Research, 71, 115-127.

84. Maher B A, Manschreck T C, Tedmon D \& Beaudette S. (1996) "Length of illness and the gradient from positive to negative semantic priming in schizophrenia patients", Schizophrenia Research, 22, 127-132.

85. Crider A. (1997) "Perseveration in Schizophrenia", Schizophrenia Bulletin, 23 (1), 63-74.

86. Chaika, E. (1995): “On analyzing schizophrenic speech: what model should we use?" en A. Sims (ed.), Speech and Language Disorders in Psychiatry, Gaskell, London.

87. Alexander, M.P., Benson, D.F., Stuss, D.T. (1989): "Frontal lobes and language", Brain and Language, 37: 656-691.

88. Andreasen, N.C. (1979b): "Thought, Language and Communication Disorders. II. Diagnostic significance", Archives of General Psychiatry, 36: 1325-1330.

89. Chantrine Y, Joanette Y, Cardebat D. (1998) "Impairments of Discourse-Level Representations and Processes", in Stemmer B \& Whitaker H A. (Eds.) Handbook of Neurolinguistics, San Diego, Academic Press.

90. Docherty N M, Schnur M \& Harvey P D. (1988) "Reference Performance and Positive and Negative Thought Disorder: A Follow-Up Study of Manics and Schizophrenics", Journal of Abnormal Psychology, Vol. 97, No. 4, 437-442.

91. Ragin A B, Oltmanns T F. (1987) "Communicability and Thought Disorder in Schizophrenics and Other Diagnostic Groups. A follow-up Study”, British Journal of Psychiatry, 150, 494-500.

92. Alverson and Rosenberg S. (1990) "Discourse analysis of schizophrenic speech: A critique and proposal", Applied Psycholinguistics 11, 167-184.

93. Dirven R \& Verspoor M. (1998) (Eds.) Cognitive Explorations of Language and Linguistics, Amsterdam/Philadelphia, John Benjamins Publishing Company.

94. Bublitz W. y Lenk U. (1999) Disturbed Coherence: 'Fill me in', en 'Coherence in Spoken and Written Discourse. How to create it and How to Describe It, editado por Wolfram Bublitz, Uta Lenk, y Elija Ventola, Amsterdam, John Benjamins Publishing.

95. Chaika, E., Lambe, R. A. (1989): "Cohesion in schizophrenic narratives, Revisited", Journal of Communication Disorders, 22: 407-421.

96. McDonald S. (1998) "Communication and Language Disturbances Following Traumatic Brain Injury", en Stemmer B \& Whitaker H A. (Eds.) Handbook of Neurolinguistics, San Diego, Academic Press. 
97. Chantrine Y, Joanette Y, Cardebat D. (1998) "Impairments of Discourse-Level Representations and Processes", in Stemmer B \& Whitaker H A. (Eds.) Handbook of Neurolinguistics, San Diego, Academic Press.

98. Schneider K. (1959) Clinical Psychopathology, New York London. Grune \& Stratton [traducido al inglés desde la Quinta Edición de Klinische Psychopathologie].

99. Harrow M and Quinlan D. (1977) “Is Disordered Thinking Unique to Schizophrenia?" Archives of General Psychiatry, 34: 15-21.

100. Harrow M, Silverstein M, Marengo J.(1983) "Disordered Thinking. Does It Identify Nuclear Schizophrenia?", Archives of General Psychiatry, 40: 765-771.

101. Marengo J T, Harrow M. (1985). "Thought Disorder. A function of Schizophrenia, Mania or Psychosis?" The Journal of Nervous and Mental Disease, Vol. 173, No.1, 35-41.

102. Grossman LS \& Harrow M. (1991) "Thought Disorder and Cognitive Processes in Mania", en Magaro P. (ed.) Cognitive Bases of Mental Disorders, Newbury Park, USA, Sage Publications.

103. Holzman P S, Shenton M E., Soloway M R. (1986) "Quality of Thought Disorder in Differential Diagnosis". Schizophrenia Bulletin, Vol. 12, 360-372.

104. Soloway, M. R., Shenton, M. E., Holzman, P. S. (1987): "Comparative studies of thought disorders. I. Mania and schizophrenia", Archives of General Psychiatry, 44: 13-20.

105. North, C. S., Hansen, K., Wetzel, R.D., Compton, W., Napier, M., Spitznagel, E.L. (1997): "Nonpsychotic thought disorder: objective clinical identification of somatization and antisocial personality in language patterns", Comprehensive Psychiatry, 38, 3:171-178.

106. O'Connell M, Cooper S, Perry C, Hoke L. (1989) "The Relationship between Thought Disorder and Psychotic Symptoms in Borderline Personality Disorder". The Journal of Nervous and Mental Disease, Vol. 117, No. 5, 273-278.

107. Braff D L and Beck A T. (1974) "Thinking Disorder in Depression". Archives of General Psychiatry, Vol. 31: 456-459.

108. Ianzito B J, Cadoret R J, Pugh D D. (1974). “Thought Disorder in Depression". American Journal of Psychiatry, 131, 6: 703-707.

109. Carter M L. (1986) "The assessment of thought Deficit in Psychotic Unipolar Depression and Chronic Paranoid Schizophrenia". The Journal of Nervous and Mental Disease, Vol. 174, No. 6, 336-341.

110. Cutting J. (1994) "The Assessment of Thought Disorder", in Barnes T R E, Nelson H E (Eds.) The Assessment of Psychoses, Oxford and Northampton, Chapman \& Hall Medical.

111. Holzman, P. S. (1995): "Thought disorders and the fundamental disturbance of schizophrenia", en R. Fog, J. Gerlach, R. Hemmingsen (eds.), Schizophrenia, Alfred Benzon Symposium 38: 409-417, Munksgaard, Copenhagen.

112. Berner P. (1992) "Vienna Research Criteria (Endogenomorphic-schizophrenic axial syndrome)", en Berner P, Gabriel E, Katschnig, Kieffer W, Koehler K, Lenz G, Nutzinger D, Schanda H, Simhandl C (Eds.) Diagnostic criteria for functional psychoses. Newcastle upon Tyne, Cambridge University Press.

113. McKenna, P. J. (1995): "The psychology of schizophrenic thought, the neuropsychology of schizophrenic speech", en A. Sims (ed.), Speech and Language Disorders in Psychiatry, Gaskell, London.

114. Nestor, P. G., Shenton, M. E., Wible, C., Hokama, H., O’Donnell, B. F., Law, S., McCarley, R. W. (1998): "Neuropsychological analysis of schizophrenic thought disorder", Schizophrenia Research, 29: 217-225. 
ORIGINALES Y REVISIONES

115. Andreasen N C (1986) "Scale for the Assessment of Thought, Language, and Communication" (TLC), Schizophrenia Bulletin, Vol. 12, No. 3, 473-482.

116. Harrow M \& Marengo T. (1986). "Schizophrenic Thought Disorder at Follow-Up: Its Persistence and Prognostic Significance". Schizophrenia Bulletin, Vol. 12, No. 3: 373- 393.

117. Peralta, V., Cuesta, M. J. y Leon, J. D. (1992): "Formal thought disorder in schizophrenia: a factor analytic study", Comprehensive Psychiatry, 33:105-110.

118. Cuesta M J, Peralta V. (1999) "Thought disorder in schizophrenia. Testing models through confirmatory factor analysis", European Archives of Psychiatry and Clinical Neuroscience, 249: 55-61.

119. Harvey, P. D., Lenzenweger, M. F., Keefe, R. S. E., Pogge, D. L., Serper, M. R., Mohs, R. C. (1992): "Empirical assessment of the factorial structure of clinical symptoms in schizophrenic patients: formal thought disorder", Psychiatry Research, 44: 141-151.

120. Kussmaul A D. (1881) Les Troubles de la Parole, Paris, Baillière et Fils.

121. Kraepelin E. (1896) "Dementia Praecox", en Cutting J y Shepherd M (ed.) The clinical roots of the schizophrenia concept, Cambridge University Press [1987].

122. Kraepelin E. (1919) 'Dementia Praecox and Paraphrenia', Edinburgh, Livingstone [la traducción inglesa procede de la octava edición en aleman del Tratado de Psiquiatria].

123. Hamilton, M. (ed.) (1984a): Fish's Schizophrenia. $3^{\mathrm{a}}$ ed., Wright, Bristol.

124. Hamilton, M. (ed.) (1984b): Fish 's Outline of psychiatry. $4^{\mathrm{a}}$ ed., Wright, Bristol.

125. Kleist, K. (1960): "Schizophrenic Symptoms and Cerebral Pathology", Journal of Mental Science, 106: 246-255.

126. Kleist, K. (1930): "Alogical thought disorder: an organic manifestation of the schizophrenic psychological deficit", en J. Cutting y M. Shepherd (ed.), The clinical roots of the schizophrenia concept, Cambridge University Press, Cambridge [1987].

127. Pearlson G D, Kreger L, Rabins P V, Chase G A, Cohen B, Wirth J B, Schlaepfer T B, Tune L E. (1989) "A chart review study of late-onset and early-onset schizophrenia". American Journal of Psychiatry, 147, 1568-1574.

128. Harvey, P.D., Lombardi, J., Leibman, M., Parrella, M., White, L., Powchik, P., Mohs, R.C., Davidson, M., Davis, K.L. (1997): "Age-related differences in formal thought disorder in chronically hospitalized schizophrenic patients: a cross-sectional study across nine decades", American Journal of Psychiatry, 154(2): 205-210.

129. Howard R, Almeida O, Levy R. (1994) "Phenomenology, demography and diagnosis in late paraphrenia". Psychological Medicine, 24 (2): 397-410.

130. Howard R, Castle D, Wessely S and Murray R. (1993) "A Comparative Study of 470 Cases of Early Onset and Late-Onset Schizophrenia". British Journal of Psychiatry, 163: 352-7.

131. Berrios, G E. (1999): "Falret, Séglas, Morselli, and Masselon, and the 'Language of the Insane': a conceptual history", Brain and Language, 69, 1: 55-75.

132. Storch A. (1924) The primitive archaic form of inner experience and thought in schizophrenia, Monograph 36, New York and Washington, Nervous and Mental Disease Publishing Company.

133. Haeckel E. (1913) The Riddle of the Universe, London, Watts \& Co (Traducido por McCabe J).

134. White W. (1926) "Language of schizophrenia". Archives of Neurology and Psychiatry, 16: 395-413.

135. Kent, G. H., Rosanoff, A. J. (1910): “A study of association in insanity”, American Journal of Insanity, 67: 317-390. 
136. Murphy, G. (1923): “Types of word association in dementia praecox, manic depressive illness, and normal persons", American Journal of Psychiatry, 2: 529-571.

137. Kasanin J S (1944) Language and Thought in Schizophrenia, New York, The Norton Library.

138. Cutting, J., Murphy, D. (1988): "Schizophrenica thought disorder. A psychological and organic interpretation", British Journal of Psychiatry, 152: 310-319.

139. Barch D M, Berenbaum H. (1995) "The categorisation of Thought Disorder", Journal of Psycholinguistic Research, Vol. 24, No. 5, 349-376.

140. Berenbaum H, Oltmanns T F \& Gottesman I I. (1988) "Indeterminacy of Heritability of Thought Disorder: Reply to Matthysse and Holzman". Journal of Abnormal Psychology, Vol. 97, No. 1, 108-109.

141. Matthysse S \& Holzman P S. (1988) "Comment on Berenbaum, Oltmanns, and Gottesman (1985): "Formal Thought Disorder in Schizophrenics and their Twins". Journal of Abnormal Psychology, Vol. 97, No. 1, 105-107.

142. Berenbaum H. (1991): "Distinguishing between content and form of speech", Archives of General Psychiatry, 48, 280.

143. Shenton, M. E., Holzman, P. S., Gale, H. J., Soloway, M. R., Coleman, M. (1991): "In reply to: Distinguishing between content and form of speech", by Berenbaum H., Archives of General Psychiatry, 48: 281-282.

144. Andreasen, N.C. (1982): "Should the term "thought disorder" be revised?" Comprehensive Psychiatry, 23, 4: 291-299.

145. Sims, A. (1988): Symptoms in the mind, Balliere Tindall, London.

146. Cox M. (1997) "The great feast of languages: passwords to the psychotic's inner world", en Mage C \& Margison F (Editores.) Psychotherapy of Psychosis, London, Gaskell.

147. Grice H P. (1975) "Logic and Conversation", in Syntax and Semantics, Vol. 3, Speech Acts, Cole P \& Morgan J L (Eds.), London, Academic Press,

148. Günther C, Maienborn C, Schopp A. (1999) "The Processing of Information Structure", en Focus. Linguistic, Cognitive, and Computational Perspectives, Peter Bosch and Rob van der Sandt (Editores), Cambridge University Press (1999).

149. Parker I, Georgaca E, Harper D, Mclaughlin T, and Stowell-Smith M. (1995) Deconstructing Psychopathology, Sage Publications, London.

150. Alpert M, Kotsaftis A, Pouget E R. (1997) "At Issue: Speech Fluency and Schizophrenic Negative Signs”, Schizophrenia Bulletin, Vol. 23, No.2, 171-177.

151. Kline P. (1993) The Handbook of Psychological Testing, Lonod, Routledge.

152. McGuirre P K, Quested D J, Spence S A, Murray R M, Frith C D, and Liddle P F. (1998) "Pathophysiology of 'positive' thought disorder in schizophrenia". British Journal of Psychiatry, 173, 231-235.

Fecha de recepción: 18/7/01

*Psiquiatra, Departamento de Psiquiatría. Universidad de Cambridge, Reino Unido.

**Neuropsiquiatra, Honorary Consultant del Addenbrooke's Hospital de Cambridge. Senior Lecturer de Psiquiatría, Departamento de Psiquiatría, Universidad de Cambridge, Reino Unido.

Correspondencia a:

Dr. G.E. Berrios, Department of Psychiatry, University of Cambridge, Addenbrooke's Hospital, Cambridge, CB2 2QQ, United Kingdom. 